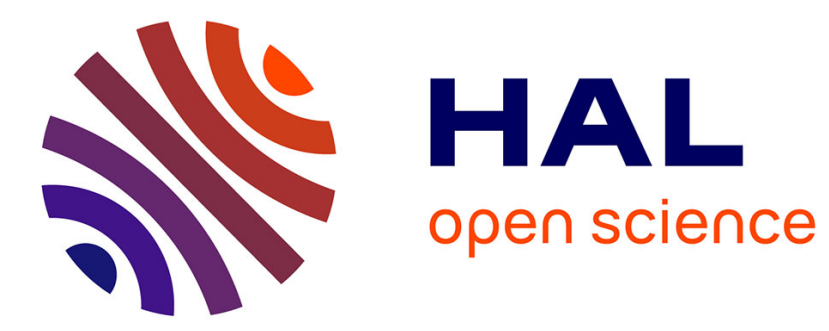

\title{
On spin glass fluctuations
}

\author{
Cirano de Dominicis, I. Kondor
}

\section{To cite this version:}

Cirano de Dominicis, I. Kondor. On spin glass fluctuations. Journal de Physique Lettres, 1984, 45 (5), pp.205-210. 10.1051/jphyslet:01984004505020500 . jpa-00232331

\section{HAL Id: jpa-00232331 https://hal.science/jpa-00232331}

Submitted on 1 Jan 1984

HAL is a multi-disciplinary open access archive for the deposit and dissemination of scientific research documents, whether they are published or not. The documents may come from teaching and research institutions in France or abroad, or from public or private research centers.
L'archive ouverte pluridisciplinaire HAL, est destinée au dépôt et à la diffusion de documents scientifiques de niveau recherche, publiés ou non, émanant des établissements d'enseignement et de recherche français ou étrangers, des laboratoires publics ou privés. 
Classification

Physics Abstracts

64.60C

\title{
On spin glass fluctuations
}

\author{
C. de Dominicis
}

Service de Physique Théorique, CEN Saclay, 91191 Gif-sur-Yvette Cedex, France

and I. Kondor

Institute for Theoretical Physics, R. Eötvös University, H 1008 Budapest, Hungary

(Reçu le 29 novembre 1983, accepté le 16 janvier 1984)

\begin{abstract}
Résumé. - Nous calculons la fonction de corrélation nue $\left\langle Q_{p}^{\alpha \beta} Q_{-p}^{\alpha \beta}\right\rangle$ pour les verres de spin à courte portée, autour de la solution de champ moyen de Parisi, en champ près de $T_{\mathrm{c}}$. Nous évaluons la corrélation associée à un recouvrement donné $x$ (des répliques $\alpha, \beta)$ et la moyenne sur $x$ i.e. la transformée de Fourier de la corrélation de spins $\overline{\left\langle\sigma_{i} \sigma_{j}\right\rangle^{2}}(p$ conjugué de $|i-j|)$. En champ le comportement infrarouge dominant est en $p^{-3}$ dans tous les cas. En champ nul il est en $p^{-4}, p^{-3}$, $p^{-3} \ln p$ respectivement pour $x \ll p, p \ll x$ et la moyenne de mécanique statistique, en accord pour le premier cas avec le résultat dynamique $(x=0)$ de Sompolinsky Zippelius.
\end{abstract}

\begin{abstract}
We compute the bare correlation function $\left\langle Q_{p}^{\alpha \beta} Q_{-p}^{\alpha \beta}\right\rangle$ for the short range spin glass, around Parisi mean field solution, in field near $T_{\mathrm{c}}$. We evaluate both the correlation function associated with a given overlap $x$ (of replicas $\alpha, \beta$ ) and the $x$-average i.e. the Fourier transform of the spin correlation $\overline{\left\langle\sigma_{i} \sigma_{j}\right\rangle^{2}}$, ( $p$ conjugate to $\left.|i-j|\right)$. In field the dominant infrared behaviour is in $p^{-3}$ in all cases. In zero field it is in $p^{-4}, p^{-3}, p^{-3} \ln p$ respectively for $x \ll p, p \ll x$ and the statistical mechanics average, agreeing in the first instance with the dynamic $(x=0)$ result of Sompolinsky Zippelius.
\end{abstract}

The present state of affairs in the field of spin glasses is to say the least, paradoxical. On the one hand some experimentalists (and theoreticians) continue to think of physical spin glasses in terms of phase transitions leaving for future developments to vindicate them. On the other hand a consensus seems to be nearing for most theoreticians and number of experimentalists on a lower critical dimension $d_{\mathrm{L}}=4$ excluding the spin glass transition from the realm of nature and rejecting it to zero temperature while shifting spin glasses to the domain of dynamical effects [1,2]. Indeed fragmentary results from various directions have long pointed towards $d_{\mathrm{L}}=4:$ high temperature expansion [3], analytic work on replica broken solutions [4], numerical simulations [5, 2]. More recently Sompolinsky and Zippelius [6] have obtained from dynamics the asymptotic behaviour of the bare fluctuation correlation in zero field conforting this trend.

This situation has motivated us to study (free) fluctuation correlations (i.e. beyond mean field approximation) in particular in the presence of a field. We have obtained in the Parisi approximation (near $T_{\mathrm{c}}$ ) the « first " propagator [4, 7] (i.e. involving only two distinct replicas) in exact form. 
For clarity we first present results on its infrared (IR) and ultra violet (UV) behaviour and compare (in zero field) with the dynamical results of Sompolinsky and Zippelius [5]. We then discuss the spectrum and the fate of zero modes. The second propagator (involving three distinct replicas) can be generated with the same methods.

\section{Mean field.}

The model used is the short-range Ising spin glass whose Hamiltonian [8] is $-\sum J_{j l} \sigma_{j} \sigma_{l}-\sum h \sigma_{j}$. The probability law $P_{j l}$ for the bonds $J_{j l}$ is a Gaussian with half width $J^{2} / z, z$ the number of (interacting) neighbours ( $z^{1 / d}$ grows like the range of the interaction). When $z$ becomes infinite like $N$ one recovers the Sherrington Kirkpatrick [9] model and mean field theory for which one has to distinguish (i) statistical mechanics and (ii) (long time) dynamical quantities. In statistical mechanics observables are computed with Parisi [10] solution (or equivalently with the ansatz proposed by Sompolinsky [11] and derived elsewhere [12]). This leads to averages over the whole replica space [13] and e.g. the standard spin glass order parameter $q$ is then

$$
q_{\mathrm{SM}} \equiv \overline{\langle\sigma\rangle_{T}^{2}}=\int_{0}^{1} \mathrm{~d} x q(x)
$$

where the bar stands for bond averaging and $q(x), x \in(0,1)$ is the Parisi order parameter function. In dynamics observables are time averages are long time behaviour is dominated by the longest relaxation time $\tau_{0}$ leading, as shown by Sompolinsky, to

$$
q_{\mathrm{Dyn}}=q\left[\tau_{0}\right]=q(x=0)
$$

for the same order parameter. A tentative explanation for this difference can be found in Houghton Jain and Young [14].

\section{Fluctuations and summary of results.}

To fix ideas we propose to compute the correlation $C_{i j} \equiv \overline{\left\langle\sigma_{i} \sigma_{j}\right\rangle^{2}}$ (or rather its Fourier transform). To keep algebra tractable we use Parisi model (quartic in the field variables $Q_{j}^{\alpha \beta}$ ) which is very good near $T_{\mathrm{c}}$. Mean field is obtained by saddle point as

$$
Q_{j}^{\alpha \beta}=\overline{\left\langle\sigma_{j}^{\alpha} \sigma_{j}^{\beta}\right\rangle} \equiv q^{\alpha \beta} .
$$

To go beyong mean field one writes (in Fourier transform, $p$ wavevector in units of the inverse range)

$$
Q_{p}^{\alpha \beta}=\sqrt{N} \delta_{p ; 0} q^{\alpha \beta}+R_{p}^{\alpha \beta}
$$

and keep terms quadratic in $R$. Free fluctuations are governed by the field Lagrangian

$$
L^{(0)}=-\frac{1}{2} \sum_{p}\left\{\sum_{(\alpha \beta)} R_{p}^{\alpha \beta}\left[p^{2}-2 \tau-2\left(q^{\alpha \beta}\right)^{2}\right] R_{-p}^{\alpha \beta}+\sum_{\alpha \neq \beta \neq \gamma} R_{p}^{\alpha \beta} q^{\beta \gamma} R_{-p}^{\gamma \alpha}\right\}
$$

where $q^{\alpha \beta}$ is given by Parisi self similar solution.

The Fourier transformed correlation in statistical mechanics is then

$$
\begin{gathered}
C_{p} \equiv 2[n(n-1)]^{-1} \sum_{(\alpha \beta)}\left\langle Q_{p}^{\alpha \beta} Q_{-p}^{\alpha \beta}\right\rangle=\delta_{p ; 0} \int_{0}^{1} q^{2}(x) \mathrm{d} x+G_{p} \\
G_{p}=2[n(n-1)]^{-1} \sum_{(\alpha \beta)}\left\langle R_{p}^{\alpha \beta} R_{-p}^{\alpha \beta}\right\rangle_{0}
\end{gathered}
$$


and $G_{p}$ is computed with $L^{(0)}$ as in (5). Note that we have

$$
G_{p}=2[n(n-1)]^{-1} \sum_{a} \mu_{a}\left[p^{2}+\lambda_{a}\right]^{-1}
$$

where $\lambda_{a}$ are eigenvalues of the quadratic form in (5) and $\mu_{a}$ the corresponding multiplicities. These eigenvalues come out in two families $[4,7,15]$ (i) the replicon one $(R)$ that produced negative masses in the early days of the theory and (ii) the longitudinal-anomalous one (LA) whose $L$ piece is studied in exact form by Thouless et al. [16] (the whole exact spectrum is given elsewhere [17, 18]). Each family has its counterpart $G_{p}^{\mathrm{R}}, G_{p}^{\mathrm{LA}}$. One also defines a correlation for a given overlap $x$ (or codistance) between replicas $\alpha$ and $\beta$, then instead of (6) one has

$$
\begin{aligned}
& C_{p}(x)=\delta_{p ; 0} q^{2}(x)+G_{p}(x) \\
& G_{p}(x)=G_{p}^{\mathrm{R}}(x)+G_{p}^{\mathrm{LA}}(x)
\end{aligned}
$$

whose integral over $x$ gives the corresponding averaged correlations $G_{p}^{\mathbf{R}}, G_{p}^{\mathbf{L A}}$. Our results are the following. For the UV behaviour (large $p$ ) one finds $G^{\mathrm{R}} \sim p^{-2} G^{\mathrm{LA}} \sim p^{-4}$. More interesting is the IR behaviour since it enters in determining $d_{\mathbf{L}}$. We have singled out from closed expressions, the behaviour of $G_{p}(x)$ for the regions $x \ll p$ and $x \gg p$, and always $p \ll 1$. This is summarized qualitatively by an interpolating form valid for all $x$ except near and above $x_{1}\left(x_{1} \sim 2 \tau\right.$ is the border of the upper flat zone in $q(x))$ where all $G_{p}{ }^{\prime}$ s behave like $p^{-2}$.

- In zero field : For the $\mathbf{R}$ family we find

$$
G_{p}^{\mathrm{R}}(x) \simeq p^{-2}\left(p^{2}+x^{2}\right)^{-1}
$$

where the limit $x=0$ agrees with Sompolinsky Zippelius [6]. For the LA family we have

$$
G_{p}^{\mathrm{LA}}(x) \simeq p^{-3}(p+x)^{-1}
$$

showing that $G_{p}^{\mathrm{LA}}(x)$ dominates the statistical average as $p \rightarrow 0$ for fixed $x$.

The respective averages contributing to $C_{p}$ become

$$
G_{p}^{\mathrm{R}} \sim p^{-3}, \quad G_{p}^{\mathrm{LA}} \sim p^{-3} \ln \left(x_{1} / p\right) .
$$

- In field : We find that $G_{p}(x, h)$ for $x \geqslant x_{0}\left(x_{0} \sim h^{2 / 3}\right.$ is the border of the lower flat zone of $q(x))$ has the same qualitative behaviour as in zero field as given in (11),(12), i.e.

$$
G_{p}(x, h) \sim[1+(x / p)](p x)^{-2} \quad x \geqslant x_{0} \gg p .
$$

The $\mathrm{R}$ family would leave Parisi's order prevail in physical space $\left(G_{p}^{\mathrm{R}} \sim(p x)^{-2}\right)$ but the IR behaviour is dominated again by $G^{\mathrm{LA}} \sim x p^{-3}$. Below $x_{0}$, the correlation remains frozen at the value $G_{p}\left(x_{0}, h\right)$. For the averages we find

$$
G_{p}^{\mathrm{R}}(h) \sim p^{-2}\left[\left(x_{0}\right)^{-1}-\left(x_{1}\right)^{-1}\right], \quad G_{p}^{\mathrm{LA}}(h) \sim p^{-3} \ln \left(x_{1} / x_{0}\right) .
$$

The effect of interactions will make $G_{p}$ more singular (if the critical exponent $\eta$ is negative [19, 20]) thus (11)-(15) tell us that $d=3$ is a lower bound for $d_{\mathrm{L}}$. When $x$ is fixed at zero, as in long time averages, the lower bound is raised to $d=4$ in zero field. This is relevant to couplings in $J_{j l}$ or $J_{j l} /\left|r_{j}-r_{l}\right|^{3}$ (that lead to an effective $\left|r_{j}-r_{l}\right|^{-6}$ potential). Note that a coupling $J_{j l} /\left|r_{j}-r_{l}\right|^{2}$ leads to replace everywhere in (11)-(15), $p^{\alpha}$ by $p^{(\alpha / 2)}$ and leaves room for a Parisi order in $d=3$. 


\section{Spectrum.}

The $R$ spectrum is given by

$$
\lambda^{\mathrm{R}}=q^{2}\left(k_{1}\right)+q^{2}\left(k_{2}\right)-2 q^{2}(\rho)
$$

with $k=k /(R+1), \rho=r /(R+1)$ where $k_{1} k_{2} r$ are three quantum numbers taking discrete values $\left(r<k_{1} \leqslant k_{2}\right)$ between 1 and $R+1$ and $R$ the number of iterations in the Parisi block ansatz (no confusion with the replicon symbol !). The $L A$ spectrum involves only one quantum number $k$, and for each $k$ contains $R+1$ modes which we label $b=1,2, \ldots, R+1$.

To understand what is going on, it is essential not to take the $R \rightarrow \infty$ limit too early. For simplicity we first discuss the L (or T.A.K. [16]) component (i.e. $k \equiv 0$ ) the eigenvalue equation of which leads to an oscillator equation. Discrete Fourier analysis of it yields a dispersion relation between the eigenvalues $\lambda^{\mathrm{L}}$ and a wavevector $u(-\pi(R+1) / 2<u<\pi(R+1))$

$$
\left(\lambda^{\mathrm{L}}\right)^{-1 / 2}=2(R+1)|\sin (u / 2(R+1))|
$$

and $u$ is quantized by $\operatorname{cotg} u x_{1}=u\left(1-x_{1}\right)$. Thus we get (i) the T.A.K. spectrum for the values of $u$ (or the index $b$ ) remaining finite as $R \rightarrow \infty$ and (ii) $\lambda^{\mathrm{L}} \sim 0\left(1 / R^{2}\right)$ for those becoming infinite with $R$ (i.e. the T.A.K. spectrum has zero measure, the zero modes constituting almost all the spectrum). Note that the T.A.K. contribution to $G_{p}$ i.e. $2[n(n-1)]^{-1} \sum_{b}\left(p^{2}+\lambda_{b}^{\mathrm{L}}\right)^{-1}$ diverges as $R \rightarrow \infty$ (and $n \rightarrow 0$ ).

Likewise the A spectrum $(k \neq 0)$ is respectively given (i) by $\lambda_{b}^{\mathrm{A}}(k) \sim 0\left(1 / R^{2}\right)$ when $b>k$ and $k$ infinite with $R$, (ii) by $q^{2}(\kappa)-q^{2}(x)$ with $x \equiv b /(R+1)$ when $b<k$ and $b$ is infinite with $R$ and finally (iii) by the non trivial spectrum described in I equations (1), (8) when $b$ remains finite as $R \rightarrow \infty$ (tending towards $q^{2}(\kappa)-q^{2}(0)$, when $b$, or the index $m$ of I, becomes infinite in turn).

\section{Multiplicities and the fate of zero modes.}

Multiplicities are best obtained by direct counting of the number of linear relationships between the eigenvectors. Once they are known they can be retrieved by setting onto its feet the direct integration over the field variables $R^{\alpha \beta}$ of $\exp L^{(0)}$ attempted by Goltsev [21, 22]. Without prior knowledge of them the direct integration is hopelessly difficult. For the LA family one gets

$$
\mu_{(k)}^{\mathrm{LA}}=n\left[\left(1 / m_{k}\right)-\left(1 / m_{k-1}\right)\right], \quad k \geqslant 1
$$

where $m_{k}$ is the number of replicas in each one of the $m_{k-1} / m_{k}$ blocks $k$ appearing at the $k$ th intermediate step of the $R$-iterated Parisi ansatz $\left(m_{0} \equiv n \rightarrow 0, m_{R+1} \equiv 1 ; m_{k} \rightarrow \kappa, m_{k}-m_{k-1} \rightarrow \mathrm{d} \kappa\right.$ as $R \rightarrow \infty)$. Again the contribution to $G_{p}$ for a given $k$ is disturbing since it blows up as $R \rightarrow \infty$ (but is now regular for $n \rightarrow 0$ ).

Fortunately this is mended by the $\mathrm{R}$ family whose multiplicity $\mu_{a}^{\mathbf{R}} \equiv \mu\left(r, k_{1}, k_{2}\right)$ is the sum of a regular and a singular part. The regular part immediately gives for the $G_{p}^{\mathbf{R}}$ contribution to (8) (after $n \rightarrow 0$ and $R \rightarrow \infty$ )

$$
G_{p}^{\mathrm{R}}=\int_{0}^{1} \mathrm{~d} \rho\left[\prod_{i=1}^{2}\left(\int_{r-0}^{1} \mathrm{~d} k_{i}\left[k_{i}^{-2}-\rho^{-1} \delta\left(k_{i}-\rho\right)\right]\right)\right]\left[p^{2}+q^{2}\left(k_{1}\right)+q^{2}\left(k_{2}\right)-2 q^{2}(\rho)\right]^{-1} .
$$

The field $h$ only enters via the order parameter $q$. Besides, for a given overlap $G_{p}^{\mathbf{R}}(x)$ is given by setting $\rho=x$ (and no $\rho$ integration) in (19) (with a simple factorized form in a " time " representation).

The singular part of $\mu^{\mathrm{R}}$ only exist for $k_{1}=r+1$. If $k_{2} \equiv k>r+1$ it is equal and opposite to $\mu^{\mathrm{LAA}}(k)$ as in (18) (its associated denominator being $p^{2}+q^{2}(\kappa)-q^{2}(\rho)$ ). If $k=r+1$, it is equal 
to $-\sum_{t=0}^{r+1} \mu^{\mathrm{LA}}(t)$ (its associated denominator being $p^{2}+0\left(1 / R^{2}\right)$ ). This singular part when combined to $G^{\mathrm{LA}}$ thus removes the $R \rightarrow \infty$ divergence to give (after a partial integration over $k$ )

$$
\begin{gathered}
G_{p}^{\mathrm{LA}}=S(1)-\int_{0}^{1} \frac{\mathrm{d} \kappa}{\kappa}\left(\frac{\mathrm{d}}{\mathrm{d} \kappa} S(\kappa)\right) \\
S(\kappa) \equiv \sum_{m=1}^{\infty}\left(\left[p^{2}+\lambda_{m}^{\mathrm{LA}}(\kappa)\right]^{-1}-\left[p^{2}+q^{2}(\kappa)-q^{2}(0)\right]^{-1}\right)
\end{gathered}
$$

now convergent. Note that $S(\kappa)$ is a constant on the flat sectors of $q(\kappa)$, and the integration only extends between $x_{0}$ and $x_{1}$. So the all pervasive zero modes have now disappeared from $G^{\mathbf{L A}}$ (except from the lower edge of the integration band). Although (20), (21) give the answer for $G_{p}^{\mathrm{LA}}$ they are difficult to use given the complicated implicit equations I (7), (8) defining $\lambda_{m}^{\text {LA }}(\kappa)$, neither they provide any clue for $G_{p}^{\mathrm{LA}}(x)$. We sketch below how to overcome these difficulties.

\section{An explicit form for $G^{\mathrm{LA}}$.}

The $(R+1)$ eigenvaluès $\lambda_{b}^{\mathrm{LA}}(k)$ for a given $k$ are given by the zeroes of the determinant read off the eigenvalue equations I (5), (6) discretized which we write $\Delta_{k} \prod_{b} X_{b}(k)$. Here $X_{b}(k)$ is $\lambda^{R}(b, b, k)$ for $b<k$ and $\lambda^{\mathbf{R}}(b, b, b)$ for $k<b$ as introduced above. Then one has

$$
S(\kappa)=\left(\partial / \partial p^{2}\right) \ln \Delta_{k} .
$$

To determine $G_{p}^{\mathrm{LA}}(x)$ one introduces $S(\kappa ; x)$ obtained by functional derivative

$$
S(\kappa ; x)=\left(\partial / \partial X_{b}(k)\right) \ln \Delta_{k}
$$

(recall $k=\kappa(R+1), b=x(R+1))$ together with, as in (20)

$$
G_{p}^{\mathrm{LA}}(x)=S(1 ; x)-x^{-1} S(x ; x)-\int_{0}^{1} \frac{\mathrm{d} \kappa}{\kappa} \frac{\mathrm{d}}{\mathrm{d} \kappa} S(\kappa ; x) .
$$

The evaluation of (23) is easily done by noticing that

$$
S(\kappa ; x) \sim \phi_{k}^{+}(x) \phi_{k}^{-}(x)\left[\Delta_{k} X_{b}^{2}(k)\right]^{-1}
$$

with $\phi$ satisfying e.g. in the simpler $k=0$ case

$$
\phi_{0}^{ \pm}(x)=\varphi^{ \pm}(x) \pm \int \frac{\mathrm{d} t}{X_{t}(0)}[q(t)-q(x)] \phi_{0}^{ \pm}(t) .
$$

Here for $\phi^{+}$we have $\varphi^{+}=q(x)$ and $(0, x)$ boundaries, for $\phi^{-}, \varphi^{-}=1$ and $(x, 1)$ [note that $\phi_{0}^{+}=q\left(x_{0}\right)$ for $x<x_{0}, \phi_{0}^{-}=1$ for $x>x_{1}$ (also in zero field $\left.\left.\phi_{0}^{-}(x=0)=\Delta_{0}\right)\right]$. Equations (24), (26) yield $G_{p}^{\mathrm{LA}}(x)$, and $G_{p}^{\mathrm{LA}}$ by $x$ integration or with (20), (22). The explicit expressions obtained would take too much space to quote. The same methods (functional derivative in $q_{b}-q_{b+1}$ ) generate the « second " propagator $[4,7]$ involving three distinct replicas.

\section{Acknowledgments.}

One of us (C.D.) would like to thank H. Sompolinsky and A. P. Young for discussions and the Institute for theoretical physics in Santa Barbara, where part of this work was performed, for its warm hospitality. 


\section{References}

[1] See e.g. Proceedings of the Heidelberg Conference (May 31st, June 2nd 1983) J. L. Van Hemmen and I. Morgenstern editors (Springer-Verlag) 1983.

[2] Binder, K., Young, A. P. UCBS preprint, 1983.

[3] Fisch, R., HARris, A. B., Phys. Rev. Lett. 38 (1977) 785.

[4] Bray, A., Moore, M. A., J. Phys. C 12 (1979) 79.

[5] Morgenstern, I., Binder, K., Phys. Rev. Lett. 43 (1979) 1615, Z. Phys. B 39 (1980) 227.

[6] Sompolinsky, H., Zippelius, A., Phys. Rev. Lett. 50 (1983) 1297, see also ref. [1].

[7] Pytte, G., Rudnick, J., Phys. Rev. B 19 (1979) 3603.

[8] Edwards, S. F., ANderson, P. W., J. Phys. F 5 (1975) 965.

[9] Sherrington, D., KirkPatrick, S., Phys. Rev. Lett. 35 (1975) 1792.

[10] Parisi, G., Phys. Rev. Lett. 43 (1979) 1574, J. Phys. A 13 (1980) 1101, 1574.

[11] SOMPOLINSKy, H., Phys. Rev. Lett. 47 (1981) 935.

[12] De Dominicis, C., Gabay, M., Orland, H., J. Physique Lett. 42 (1981) L-523.

[13] De Dominicis, C., Yourng, A. P., J. Phys. A 16 (1983) 1063.

[14] Houghton, A., Jain, S., Young, A. P., J. Phys. A 16 (1983) L-375.

[15] De Almeida, J. R. L., Thouless, D. J., J. Phys. A 11 (1978) 983.

[16] Thouless, D. J., De Almeida, J. R. L., Kosterlitz, J. M., J. Phys. C 13 (1980) 3272.

[17] De Dominicis, C., Kondor, I., Phys. Rev. B 27 (1983) 606 hereafter referred as I.

[18] Kondor, I., De Dominicis, C., J. Phys. A 16 (1983) L-73.

[19] Chen, J. H., Lubensky, T. C., Phys. Rev. B 16 (1977) 2106.

[20] ZIPPELIUS, A., JÜLICH, preprint 1983, see also ref. [1].

[21] Goltsev, A. V., J. Phys. A 16 (1983) 1337.

[22] Kondor, I., De Dominicis, C., J. Phys. A 16 (1983) 3931. 